\title{
Expression of a homeobox gene (SIX5) in borderline ovarian tumours
}

\author{
C Winchester, S Robertson, T MacLeod, K Johnson, M Thomas
}

\begin{abstract}
Aims-To assess the expression of SIX5 (a homeobox gene) mRNA in surface coelomic epithelium, endocervical epithelium, Fallopian tube epithelium, and benign, borderline, and malignant epithelial ovarian tumours.

Methods-10 normal premenopausal ovaries, 10 normal Fallopian tubes, 10 normal cervices, 10 normal postmenopausal ovaries, 10 benign epithelial ovarian tumours, 10 malignant epithelial ovarian tumours, and 40 borderline epithelial ovarian tumours were studied retrospectively. The tissues had been fixed in formalin and embedded in paraffin wax. The tumours had previously been typed into mucinous, serous, or mixed tumours and assigned to the borderline category according to the FIGO/WHO criteria. Expression was assessed by in situ binding of SIX 5 specific sense and antisense riboprobes. Hybridisation of the riboprobes was detected using a standard immunohistochemical technique and the results correlated with expression in the normal epithelium of the endocervix, Fallopian tube, surface coe-
\end{abstract} lomic epithelium, and ovarian tumours. Results-Expression of SIX 5 mRNA was demonstrated in normal Fallopian tube epithelium and normal endocervical epithelium. SIX 5 mRNA was not detected in normal ovarian epithelial tissue at any of the times studied during the menstrual cycle. Expression of SIX5 was not shown in benign epithelial ovarian tumours or in any of the malignant epithelial ovarian tumours. In 31 of 37 borderline epithelial ovarian tumours (84\%), SIX5 expression was found in the epithelial cells.

Conclusions-SIX5 expression is present in the normal epithelium throughout most of the female reproductive tract, suggesting it may have a role in maintaining epithelial differentiation in these tissues. SIX5 expression appears to be restricted to borderline epithelial ovarian tumours and may be a marker of epithelial differentiation in these tumours; thus borderline ovarian tumours may not be part of a continuum of disease between benign and malignant epithelial ovarian tumours. Further investigation of expression of SIX5 may clarify the molecular processes that promote differentiation of the ovarian surface epithelium.

(f Clin Pathol 2000;53:212-217)

Keywords: ovarian epithelial tumours; SIX5; homeobox genes
There is considerable debate about the natural history of the development of malignant epithelial ovarian tumours. This arises because of difficulties in accessing ovarian tissue and because of the poor correlation between clinical evaluation at laparoscopy and histology, ${ }^{1-4}$ and between ultrasound appearances and histology. It is well established that early stage ovarian cancer has a much better five year survival than advanced presentation disease $(80 \% v 35 \%)$, but the difficulty lies in the detection of early malignant or premalignant changes in the ovary. It has been suggested that borderline epithelial ovarian tumours are part of a continuum of progressive change from benign to malignant, ${ }^{5}$ showing some features of malignancy in the epithelial cells but without stromal invasion (as defined by the FIGO/WHO criteria). ${ }^{67}$ The epidemiological evidence suggests a link between borderline and malignant epithelial ovarian tumours, with age at first childbirth, parity, contraceptive pill use, and postmenopausal status being correlated with the development of both types of tumour. ${ }^{8}$ The incidence of both tumour types is increasing, although borderline epithelial ovarian tumours remain much less prevalent than malignant epithelial ovarian tumours, with incidences of 4.8/100 000 person-years and 14/100 000 person-years, respectively, in Norway. ${ }^{9}$

Detailed knowledge of the pathogenesis of ovarian tumours, and in particular the timing of progression of the disease, would help the detection of early stage disease and optimise the timing of surgical or chemotherapeutic interventions. Advances in molecular techniques have allowed us to determine genetic variations between different types of tumour and the sequences of molecular events that lead to malignant change. In the case of the ovary there is conflicting molecular evidence about the genesis of malignant epithelial ovarian tumours and where or whether borderline epithelial ovarian tumours fit in. Studies of ovarian tumours have concentrated on molecular changes known to occur in other malignancies such as those affecting oncogenes, tumour suppressor genes, and programmed cell death signallers such as p53. The differentiation from totipotential to specialised epithelial cells is common to benign, borderline, and malignant epithelial ovarian tumours and suggests that genes important in initiating patterned differentiation may be a molecular trigger common to the range of epithelial ovarian tumours. Homeobox genes are good candidates for triggering the molecular changes that may be involved in patterns of differentiation 
and proliferation in embryonic tissues and in maintaining patterning and differentiation in adult tissues. Recent studies have shown homeobox gene involvement in leukaemias ${ }^{10}$ in solid tumours such as breast, ${ }^{11}$ kidney, lung, and colon. ${ }^{12}$

The aim of this study was to determine the expression of a homeobox gene (SIX5) in the ovary in surface coelomic epithelium, benign epithelial ovarian tumours, borderline epithe-
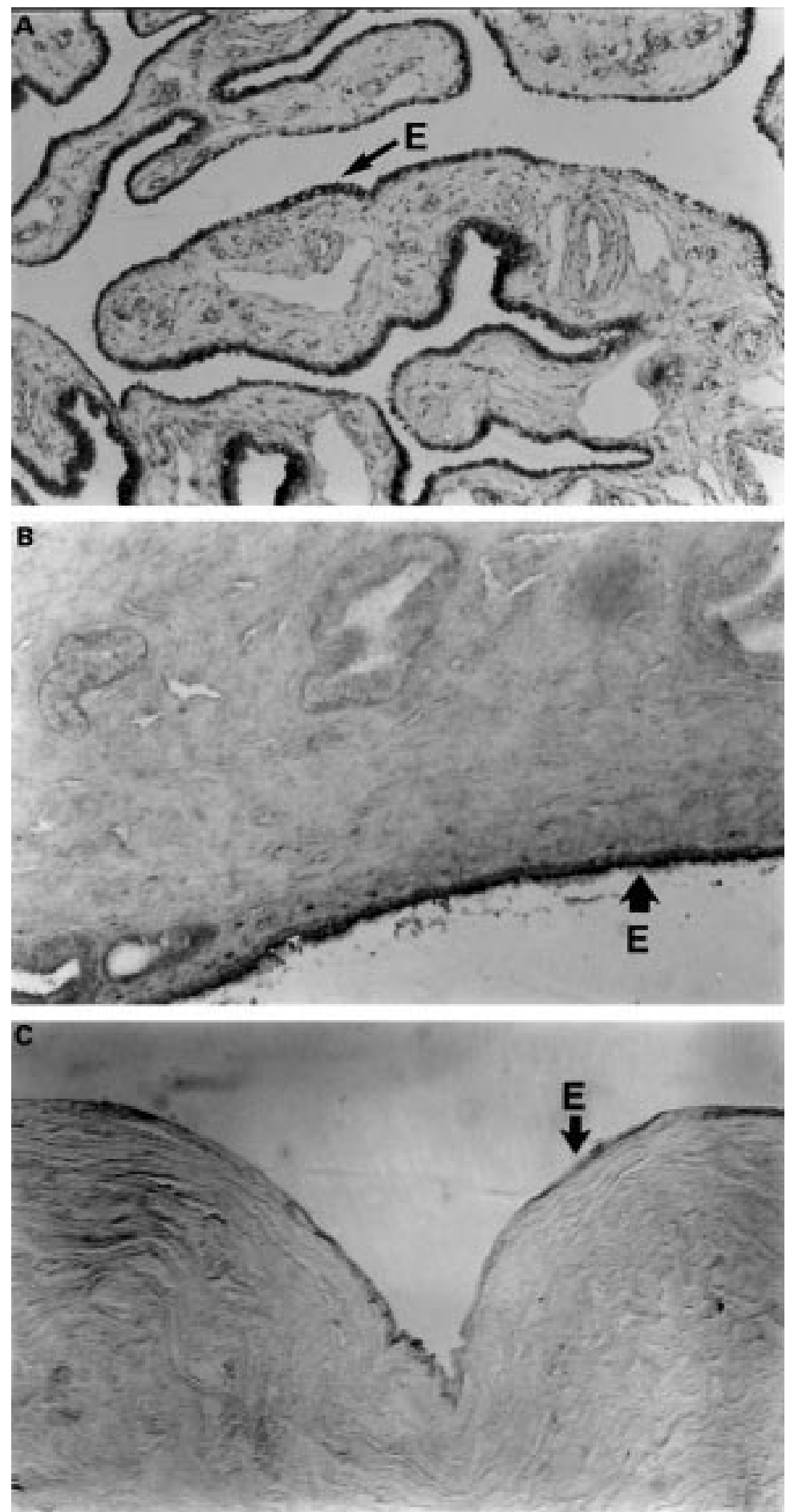

Figure 1 Analysis of SIX5 expression in (A) normal Fallopian tube showing strong expression in the epithelium (×65); (B) normal endocervix demonstrating strong expression in the endocervical epithelium, but not in the deeper glandular epithelium ( $\times 65) ;(C)$ normal ovary ( $\times 65$, oblique illumination) showing no staining in the epithelium. Epithelial layers are marked $E$. lium, and malignant epithelium. It was hoped that by analysing changes in the expression in the different epithelia, the relation between the different types of ovarian tumour could be assessed and any association between them defined.

\section{Methods}

TISSUE SAMPLES

Ten normal premenopausal ovaries, 10 normal postmenopausal ovaries, 10 benign ovarian tumours, 37 borderline epithelial ovarian tumours, and 10 malignant epithelial ovarian tumours were studied retrospectively. The normal ovaries were collected at different stages throughout the menstrual cycle, as ascertained by the date of the last menstrual period (LMP). Normal ovarian tissue was obtained from women undergoing hysterectomy for gynaecological procedures unrelated to potential ovarian pathology. The benign ovarian tumours were obtained from women undergoing oophorectomy at the time of hysterectomy and undergoing oophorectomy for tissue diagnosis of an ovarian mass. Five of the cysts studied were inclusion cysts with serous epithelium close to the coelomic surface of the ovary. Two were serous cystadenomas and three were mucinous cystadenomas. The borderline tumours were assessed according to the WHO/ Figo criteria ${ }^{67}$ and represented serous, mucinous, and mixed serous and mucinous types, stage 1A-1C. Only blocks from the tumours themselves (and not implants) were used for the study. Nineteen $(51 \%)$ were mucinous cystadenomas, $16(43 \%)$ were serous cystadenomas, and two (5.4\%) showed features of both serous and mucinous cystadenomas. Five serous cystadenocarcinomas FIGO stage III and five mucinous cystadenocarcinomas FIGO stage III were studied. All the tissues had been fixed in formalin and embedded in paraffin wax. The ovaries were examined after staining with haematoxylin and eosin. Only ovaries that had intact surface coelomic epithelium over the ovarian surface were used. The fragile nature of the epithelium meant that many specimens were denuded of epithelium during handling and preparation and therefore were not suitable for analysis. Control sections of formalin fixed, wax embedded tissue from normal Fallopian tube and normal cervix were analysed to ascertain whether serous and mucinous differentiated cells in these tissues showed expression of SIX 5.

The normal ovarian tissue, normal cervical tissue, Fallopian tubes, benign epithelial ovarian tumours, malignant epithelial ovarian tumours, and 21 borderline epithelial ovarian tumours were obtained from the archive of the pathology laboratory, Western Infirmary, Glasgow, and 16 borderline epithelial ovarian tumours were obtained from the pathology laboratory, Glasgow Royal Infirmary.

IN SITU HYBRIDISATION

Sections were cut at $4 \mu \mathrm{m}$ thickness from paraffin blocks and mounted onto APES coated slides. The sections were processed according to a standard protocol ${ }^{13}$ and 


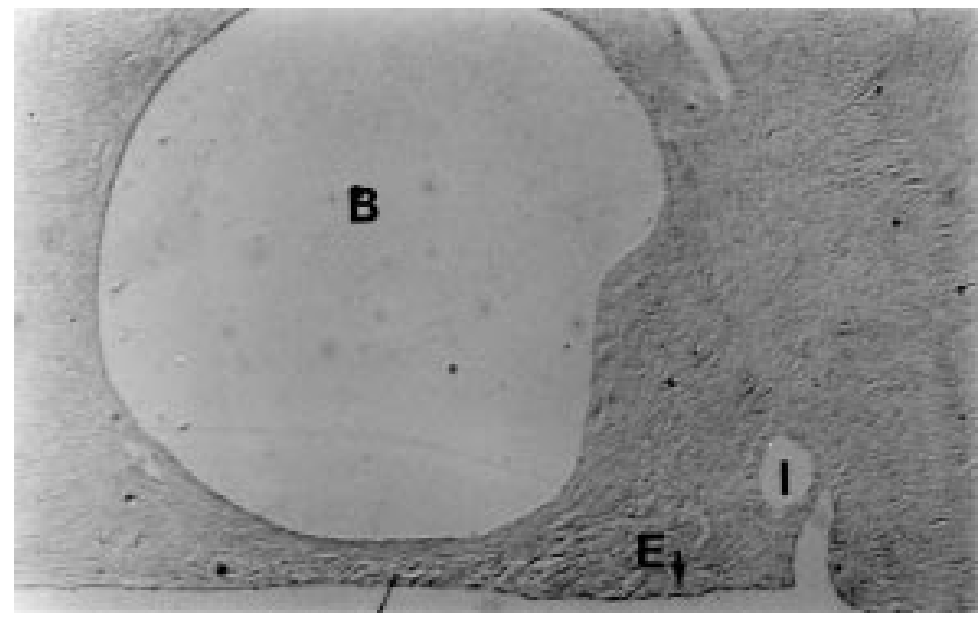

Figure 2 Analysis of coelomic surface epithelium (E), inclusion cyst epithelium (I), and benign cyst epithelium (B) (×33). None of the epithelia show expression of SIX5.

hybridised with digoxigenin (Boehringer Mannheim) labelled sense and antisense SIX5 riboprobes. Digoxigenin labelled sense and antisense SIX 5 riboprobes were generated by in vitro transcription of pSIX5.1. The SIX5 riboprobe generated was designed to specifically detect only SIX 5 mRNA, as it contains sequence mainly from the 5'UTR and does not include any regions that are similar to other human SIX genes.

The SIX5 subclone, pSIX5.1, was generated by first amplifying genomic DNA using a forward primer in the 3'UTR of DMPK, FAS (5' TCC TCA CTG CGC TGC TCT C 3') and a reverse primer in exon A of SIX5, RAS (5' TGC GCA GTC GAT ACT TGT CCA C $\left.3^{\prime}\right)$. The PCR product was subcloned into the $\mathrm{T}$ vector pMOSBlue (Amersham International) and then cut with EcoRI-BamHI to release a SIX 5 fragment of 652 base pairs (bp) (662-1314 of accession number X84813). The 652 bp SIX 5 fragment was ligated unidirectionally into EcoRI-BamHI cut pBluescript ${ }^{\mathbb{B}}$ $\mathrm{SK}(+)$, to create pSIX 5.1. The SIX5 sense riboprobe was generated by linearising pSIX 5.1 with BamHI and transcribing with T7 RNA polymerase (Promega), and the SIX5 antisense riboprobe was generated by linearising pSIX5.1 with EcoRI and transcribing with T3 RNA polymerase (Promega).

One microgram of each template was linearised and the DNA transcribed with 40 units of T7 or T3 RNA polymerase (Promega) at $3^{\circ} \mathrm{C}$ for four hours. The reaction mix contained $10 \mathrm{mM}$ dithiothreitol (DTT), 20

Table 1 Expression of SIX5 mRNA in ovarian epithelium

\begin{tabular}{llc}
\hline & \multicolumn{2}{l}{ SIX5 expression } \\
\cline { 2 - 3 } Tissue type & Positive & Negative \\
\hline Normal follicular phase ovary & 0 & 4 \\
Normal midcycle ovary & 0 & 2 \\
Normal secretory phase ovary & 0 & 4 \\
Normal postmenopausal ovary & 0 & 10 \\
Malignant EOTs & 0 & 10 \\
Borderline serous EOTs & $16(13$ stage 1A; 2 stage 1B; & 0 \\
Borderline mucinous EOTs & 1 stage 1C) & 5 (stage 1A) \\
Borderline mixed serous and mucinous EOTs & 1 (stage 1A) & 1 (stage 1A) \\
\hline
\end{tabular}

EOT, epithelial ovarian tumour. units of RNasin (RNase inhibitor) (Promega), $1 \times$ transcription buffer (Promega), $1 \mathrm{mM}$ of adenosine triphosphate (ATP), $1 \mathrm{mM}$ of cytosine triphosphate (CTP), $1 \mathrm{mM}$ of guanosine triphosphate (GTP), $0.65 \mathrm{mM}$ of uridine triphosphate (UTP), and $0.35 \mathrm{mM}$ digoxigenin-11-UTP (Boehringer Mannheim). The riboprobes were precipitated and resuspended in $40 \mu \mathrm{l}$ of DEPC treated water.

The probe sequence was checked by comparing it with the sequence of all known human SIX genes and all sequences in the DNA and protein databases. It did not match any sequence apart from SIX5. The specificity of the riboprobe interaction was checked for in situ hybridisation experiments of other human tissue. ${ }^{13}$ The specificity of the staining was checked by RNase treatment before and after hybridisation with the riboprobe and confirmed that the SIX5 antisense riboprobe was specifically interacting with mRNA.

In control experiments, sections were treated with $100 \mathrm{mg} / \mathrm{ml} \mathrm{RNase} A$ at $37^{\circ} \mathrm{C}$ for $30 \mathrm{~min}$ utes before the prehybridisation step or for 30 minutes after the hybridisation step; with hybridisation buffer without riboprobe; or by hybridisation with sense riboprobe.

The interactions were detected colorimetrically (blue/black) with alkaline phosphatase substrates $(0.33 \mathrm{mg} / \mathrm{ml} \mathrm{NBT}$ (nitroblue tetrazolium salt in $70 \% \mathrm{vol} / \mathrm{vol}$ dimethyl formamide) (Sigma), $0.17 \mathrm{mg} / \mathrm{ml} \mathrm{BCIP} \mathrm{(5-bromo-4-}$ chloro-3-indolylphosphate toluidinium salt in $100 \%$ dimethyl formamide) (Sigma), and 0.23 $\mathrm{mg} / \mathrm{ml}$ levamisole (Sigma) at room temperature.

\section{PHOTOMICROGRAPHS}

Photomicrographs were taken of the sections on an Olympus BX 50 microscope on Kodak ectochrome $64 \mathrm{~T}$ film. Oblique illumination was produced by introducing a flat opaque object into the condenser.

\section{Results}

SIX5 expression was demonstrated in normal surface endocervical epithelium and normal Fallopian tube epithelium, as demonstrated in fig 1. Staining was strong and homogeneous within the epithelia of these tissues. The glandular epithelium of the cervix did not appear to express SIX5.

The 10 normal premenopausal ovaries were obtained at various stages of the menstrual cycle. Four were collected in the early follicular phase, two at mid cycle, and four in the secretory phase according to LMP dates. Expression of SIX5 was not demonstrated, either in normal premenopausal ovarian epithelial tissue (as shown in fig 1) at any time during the menstrual cycle sampled in our study, or in postmenopausal ovarian epithelial tissue. Expression of SIX 5 was not shown in benign epithelial ovarian tumours or inclusion cyst epithelium (fig 2) or in any of the malignant epithelial ovarian tumours.

In 31 of 37 borderline tumours ( $85 \%$ ), focal SIX5 expression was found in the epithelial cells. The distribution and intensity of the staining did not correlate with morphological 

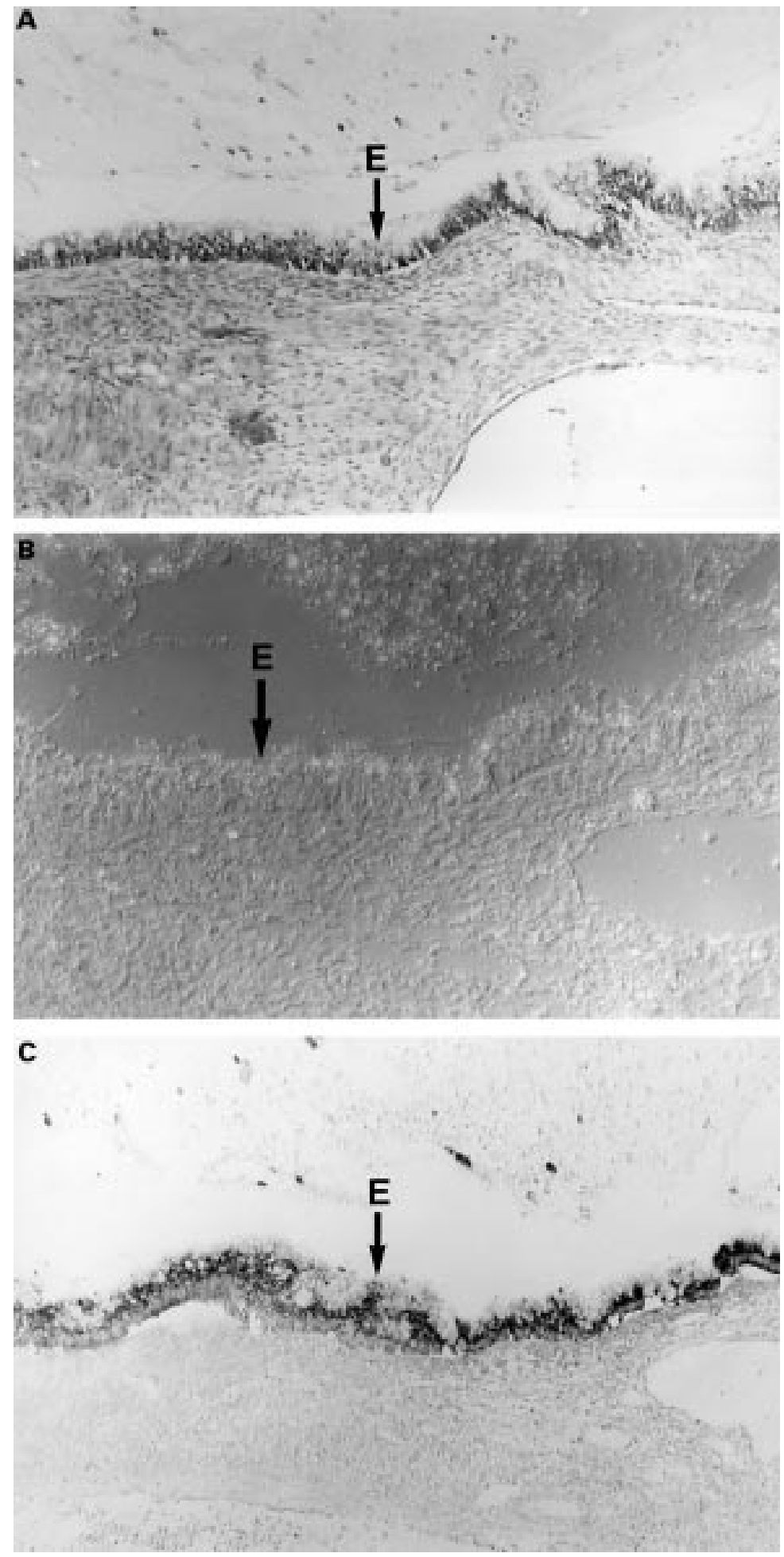

Figure 3 Analysis of SIX5 $m$ RNA expression in serial sections of a borderline epithelial ovarian tumour. (A) Stained with haematoxylin and eosin $(\times 100)$; (B) hybridised with sense SIX5 riboprobe (control) ( $\times 100$ oblique illumination); $(C)$ hybridised with antisense SIX5 riboprobe ( $\times 100$ oblique illumination) showing expression in the epithelial cell layer. Epithelial layers are marked $E$.

changes in the epithelial cells in different regions of the borderline tumours. Control sections hybridised with sense riboprobe showed less or no staining in most cases. In 10 cases, staining with the sense riboprobe was as intense as that seen with the antisense probe. Treatment with RNase A before hybridisation decreased staining in all the antisense positive tissues, while treatment after hybridisation failed to alter the intensity of staining, indicating that the antisense riboprobe was specifically binding to SIX5 mRNA. All the positive borderline epithelial ovarian tumours showed long stretches of epithelium, with intense staining in the perinuclear region and cytoplasm of the cells. A summary of these results is shown in table 1 and examples of expression of SIX5 and the sense riboprobe control are shown in fig 2 . There was variation in distribution and intensity of staining in different areas of the epithelium as demonstrated in fig 3. The same pattern of variation was seen in labelled serial sections suggesting that this reflected a true difference in expression and was not an artefact of the labelling procedure.

\section{Discussion}

The aim of molecular studies in ovarian epithelial disease is to develop a detailed understanding of tumour progression akin to the paradigm of bowel cancer. The discovery of mutations in BRCA1 and BRCA2 genes has allowed identification of some individuals at high risk with a strong family history of ovarian epithelial cancer, but there are no published data showing an increase in both borderline and malignant epithelial ovarian tumours which would lend support to a common molecular pathway in their genesis.

Changes in microsatellite repeat number have been shown in borderline and early ovarian tumours and some advanced tumours. ${ }^{14-16}$ In individual women with mismatch repair gene mutations (which lead to widespread microsatellite instability), a $10 \%$ incidence of malignant epithelial ovarian tumours can be anticipated. ${ }^{16}$ SIX5 is a homeobox gene situated adjacent to a well known site of repeat instability, a (CTG)n repeat on chromosome 19. ${ }^{17}$ Expansion of this (CTG)n repeat is associated with the development of myotonic dystrophy. A change in repeat number at the DM locus has been found in breast tumours. ${ }^{18}$ In a previous study we have not observed (CTG)n repeat expansion at this site in DNA extracted from a variety of benign and malignant ovarian tumours compared with normal tissue DNA (Thomas M, unpublished data). SIX5 is a member of the SIX gene family and encodes a homeodomain transcription factor protein capable of altering gene expression at other sites in the genome. All the SIX genes are characterised by two functionally important regions, the SIX box and the homeobox, from which the name was derived. Reverse transcriptase polymerase chain reaction (RT-PCR) and northern blot analyses have shown widespread expression of SIX 5 in fetal and adult tissues. ${ }^{17}$ Localisation of SIX5 mRNA to specific cell types has only been characterised in the eye, where SIX5 was shown to be expressed in epithelial cells of various eye structures. ${ }^{13}$

Previous work on ovarian pathology has not shown consistent sequential changes in the genome analogous to those seen in bowel tumours. Molecular changes have been described in both borderline epithelial ovarian 


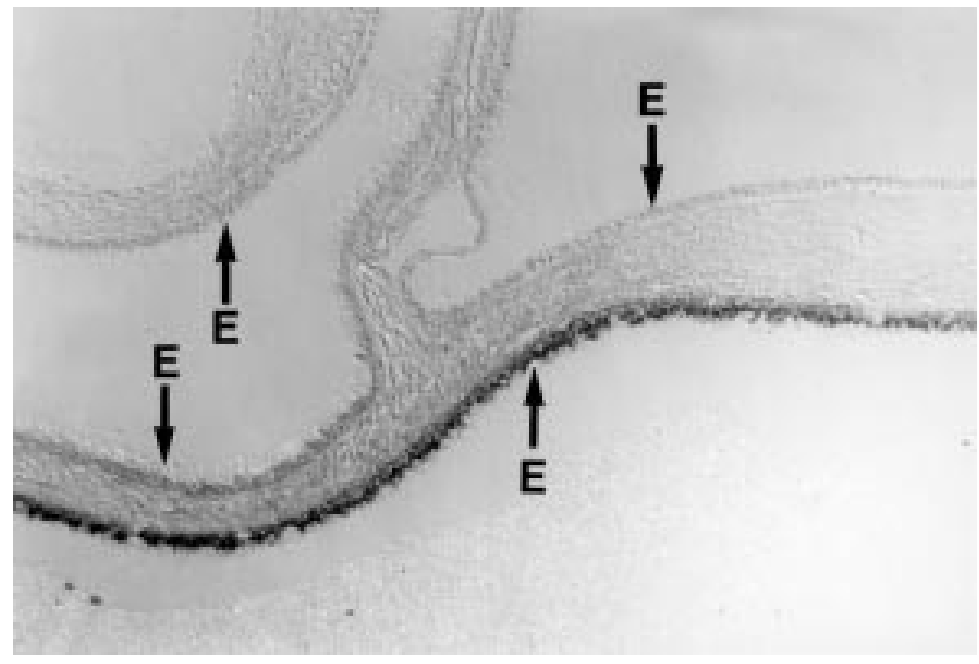

Figure 4 Variation in SIX5 expression in a stage IA serous borderline epithelial ovarian tumour, showing the focal nature of expression in the epithelium of some cysts but not others ( $\times 65$, oblique illumination). artefact remains, this was not seen in borderline tumours such as in fig 4 , where some edges were clearly positive and others negative. Further work on microdissected regions showing focal and no staining of the mRNA message would determine whether there is a quantitative change associated with these observations. The lack of hybridisation in the surface coelomic epithelium and malignant tumour samples does not mean that there is no expression of SIX5. In situ hybridisation may not be sufficiently sensitive to detect the small amounts of mRNA production that may be required to maintain patterning in an already differentiated tissue. Indeed, RT-PCR studies on normal whole ovary have shown SIX 5 mRNA expression, although it is not possible to say which cell types within the ovary were contributing to the mRNA detected (Winchester $\mathrm{C}$, unpublished data).

There are two different types of ovarian mucinous tumour of borderline malignancy. In most cases, the mucinous epithelium is of intestinal type, but in a minority it is of endocervical type. ${ }^{23}{ }^{24}$ It has been shown that this morphology correlates with differences in amylase production in the tumour cells and these differences are also observed in normal bowel and endocervical epithelium. ${ }^{25}$ All the mucinous borderline epithelial ovarian tumours in this study were morphologically classified as intestinal epithelial type and were stage 1A. If the role of SIX 5 was confined to the female genital tract, it may have been anticipated that only serous tumours would show expression of SIX5. This was not the case, as SIX5 expression was present in both serous and mucinous borderline epithelial ovarian tumours (although all the borderline tumours that failed to show expression were of the mucinous type).

The lack of expression in the epithelium of premenopausal ovaries at various stages in the menstrual cycle, in postmenopausal ovaries, and in benign and malignant epithelial ovarian tumours suggests that borderline epithelial ovarian tumours are not part of a continuum of disease between benign and malignant tumours. However, our findings do not rule out this possibility. Transient overexpression of homeobox genes in bowel epithelium has suggested a regulatory link between the HOXB8 and several tumour suppressor genes such as DCC, APC, and TGF $\beta .^{26}$ Overexpression of another SIX gene (SIX1) has been found in breast tumours in association with abrogation of the G2 cell cycle check point. This is of particular interest as the epidemiology of and the genetic (BRCA1 and BRCA2) predisposition to breast and ovarian cancer are very similar. It is possible that the SIX genes may have a regulatory link with BRCA1 and BRCA2.

The expression of SIX5 in borderline epithelial ovarian tumours shows that a molecular change has occurred in differentiated epithelial cells and suggests its potential as a molecular trigger of pathogenesis in most borderline epithelial ovarian tumours. Further investigation by microdissection studies of adjacent and contralateral benign, borderline, deeper glandular epithelium did not. This phenomenon was present in all the cervices studied and although the possibility of an edge 
and malignant epithelial ovarian tumours and determination of the links with genes involved in cell proliferation are needed to clarify the relation between benign, borderline, and malignant ovarian epithelial change.

We thank Dr C Stewart, Prof I T Cameron, and Prof S Campo for their help and guidance compiling this manuscript and $\mathrm{Mr} \mathrm{R}$ Ferrier for his technical assistance. KJ and CW are supported by the Muscular Dystrophy Group of Great Britain and Northern Ireland and SR by the Hall Fund, University of Glasgow.

1 Nicoloso E, d'Ercole C, Boubli L, et al. Borderline tumors and cancers of the ovary. Presse Med 1995;24:1421-4.

2 Ganjei P, Dickinson B, Harrison T, et al. Aspiration cytology of neoplastic and non-neoplastic ovarian cysts: is it of neoplastic Int f Gynecol Pathol 1996;15:94-101.

3 Gerber B, Gustmann G, Kulz T, et al. Histology and cytology of laparoscopically operated "simple ovarian cysts". ogy of laparoscopically operated "simple

4 Blanc B, Boubli L, D'Ercole C, et al. Laparoscopic management of malignant ovarian cysts: a 78-case national survey. Part 1. Pre-operative and laparoscopic evaluation. Eur $\mathcal{F}$ Obstet Gynecol Reprod Biol 1994;56:177-80.

5 Matias-Guiu X, Prat J. Molecular pathology of ovarian carcinomas. Virchows Arch 1998;433:103-11.

6 International Federation of Gynecology and Obstetrics, classification and staging of malignant tumours in the female pelvis. Acta Obstet Gynecol Scand 1971;50:1-7.

7 Serov SF, Scully RE, Sobin LH. International histological classification and staging of tumours. In: Histologic typing of ovarian tumours. Geneva: World Health Organisation, 1973.

8 Adami HO, Hsieh CC, Lambe M, et al. Parity, age at first childbirth and risk of ovarian cancer. Lancet 1994;344: 1250-4.

9 Bjorge T, Engeland A, Hansen S, et al. Trends in the Bjorge T, Engeland A, Hansen S, et al. Trends in the incidence of ovarian cancer and borway. Int $\mathcal{F}$ Cancer 1998;71:780-6.

10 Lawrence HJ, Sauvageau G, Humphries RK, et al. The role of HOX homeobox genes in normal and leukemic haematopoiesis. Stem Cells 1996;14:281-91.

11 Ford HL, Kabingu EN, Bump EA, et al. Abrogation of the G2 cell cycle checkpoint associated with overexpression of HSIX1: a possible mechanism of breast carcinogenesis. Proc Natl Acad Sci USA 1998;95:12608-13.
12 Cillo C. Apropos of the studies of Lewis, Nusslein-Volhard and Wieschaus, 1995 Nobel prize winners, on the genetic mechanisms of embryonic development of drosophila. A model for human cancer progression. Bull Cancer 1996;83:

3 Winchester CL, Ferrier RK, Sermoni A, et al. Characterization of the expression of DMPK and SIX5 in the human eye and implications for pathogenesis in myotonic dystrophy. Hum Mol Genet 1999;8:481-92.

14 Lynch HT, Casey MJ, Lynch J, et al. Genetics and ovarian carcinoma. Semin Oncol 1998;25:265-80.

15 King BL, Carcangiu M-L, Carter D, et al. Microsatellite instability in ovarian neoplasms Br f Cancer 1995;72:37682.

16 Tangir J, Loughridge NS, Berkowitz RS, et al. Frequent microsatellite instability in epithelial borderline ovarian tumours. Cancer Res 1996;56:2501-5.

17 Boucher CA, King SK, Carey N, et al. A novel homeodomain encoding gene is associated with a large $\mathrm{CpG}$ island interrupted by the myotonic dystrophy unstable (CTG)n repeat. Hum Mol Genet 1995;4:1919-25.

18 Shaw JA, Walsh T, Cappell SA, et al. Microsatellite instability in early sporadic breast cancer. Br f Cancer 1996;73: 1393-7.

19 Berchuck A, Carney M. Human ovarian cancer of the surface epithelium. Biochem Pharmacol 1997;54:541-4.

20 Mok SC, Bell DA, Knapp RC, et al. Mutation of K-ras protooncogene in human epithelial ovarian tumours of borderline malignancy. Cancer Res 1993;53:1489-92.

21 Hutson R, Ramsdale J, Weels M. P53 protein expression in putative precursor lesions of epithelial ovarian cancer. Histopathology 1995;27:367-71.

22 Katso RM, Manek S, O’Byrne K, et al. Molecular approaches to diagnosis and management of ovarian cancer. Cancer Metastasis Rev 1997;16:81-107.

23 Rutgers JL, Scully RE. Ovarian Mullerian mucinous papillary cystadenomas of borderline malignancy: a clinicopathologic analysis. Cancer 1988;61:340-8.

24 Szymanska K, Szamborski J, Miechowieka N, et al. Malignant transformation of mucinous ovarian cystadenomas of intestinal epithelial type. Histopathology 1983;4: mas of 197 . 09 .

25 Bruns DE, Mills SE, Savory J. Amylase in fallopian tube and serous ovarian neoplasms: Immunohistochemical localisation. Arch Pathol Lab Med 1982;106:17-20.

26 Vider BZ, Zimber A, Hirsch D, et al. Human colorectal carcinogenesis is associated with deregulation of homeobox gene expression. Biochem Biophys Res Commun 1997;232: $742-8$. 